\title{
ABORDAGEM FISIOTERAPÊUTICA PELO CONCEITO NEUROEVOLUTIVO BOBATH NA SÍNDROME CRI DU CHAT: ESTUDO DE CASO
}

\author{
Amanda Rodrigues de Oliveira, Larissa Bianchi Ilhéu, Fabiana Nonino de Sá \\ UNICESUMAR \\ amandaroliveirs@gmail.com
}

Acesso DOI: http://dx.doi.org/10.34059/ciejop.2019v27i1-4

\section{Resumo}

A Síndrome Cri Du Chat (SCDC) é uma doença genética que resulta de uma deleção extremamente pequena (5p15.2) ou uma deleção de todo o braço curto do cromossomo (5p). É uma doença rara, com incidência de $1 / 15000$ a $1 / 50000$ nascidos vivos, tendo prevalência maior no sexo feminino. As manifestações clínicas caracterizam-se por choro típico, conhecido como miado de gato, desvio inferior das extremidades dos lábios, face arredondada, altura e peso inferior aos padrões, ponte nasal ampla, dobras epicanas, orelhas com baixa implantação, hipotonia, hipertelorismo, microretrognatismo e atraso do desenvolvimento psicomotor. $O$ tratamento baseia-se na reabilitação precoce, em que a fisioterapia atua na prevenção das complicações e na correção das deformidades. Desse modo, o objetivo deste trabalho foi verificar a eficácia da contribuição do Conceito Bobath no tratamento da paciente com SCDC. O estudo de caso foi feito com uma criança, do sexo feminino, 4 anos de idade, com diagnóstico confirmado por exame genético. A paciente foi submetida à avaliação através dos protocolos Teste Denver e Inventário de Avaliação Pediátrica de Incapacidade (PEDI). A intervenção foi feita em 20 sessões, na clínica de fisioterapia da ANPR em Maringá-PR, durante 30 minutos, com repetições de 2 vezes por semana, totalizando 2 meses de tratamento, através do Conceito Bobath. Foi realizada estimulação motora na paciente, de acordo com suas necessidades. Os resultados encontrados mostram que o tratamento através da abordagem fisioterapêutica, utilizando o Conceito Bobath, pode aprimorar o desenvolvimento motor da criança, promovendo independência da mesma.

Palavras-chave: fisioterapia, genética, desempenho psicomotor.

\section{Abstract}

Cri du Chat Syndrome (CDCS) is a genetic disease that results from an extremely small deletion (5p15.2) or a deletion of the entire short arm of the chromosome (5p). It is a rare disease, with an incidence of $1 / 15000$ to $1 / 50000$ live births, with a higher prevalence in females. The clinical manifestations are characterized by typical crying, known as meowing of cat, inferior deviation of the extremities of the lips, rounded 
face, height and weight below the standards, wide nasal bridge, epicanthic folds, ears with low implantation, hypotonia, hypertelorism, microretrognatism and delayed psycho-motor development. The treatment is based on early rehabilitation, in which physiotherapy works to prevent complications and to correct deformities. Thus, the objective of this study was to verify the efficacy of the Bobath Concept contribution in the treatment of patients with CDCS. The case study was done with a 4-year-old female child with a confirmed diagnosis by genetic screening. The patient was submitted to the evaluation through the Denver Test and Pediatric Disability Assessment (PEDI) protocols. The intervention was performed in 20 sessions at the physical therapy clinic of ANPR in Maringá-PR, for 30 minutes, with repetitions of 2 times a week, totaling 2 months of treatment, through the Bobath Concept. Motor stimulation was performed in the patient according to their needs. The results show that the treatment through the physiotherapeutic approach, using the Bobath Concept can improve the child's motor development, promoting her independence. Keywords: physical therapy specialty, genetics, psychomotor performance.

\section{INTRODUÇÃO}

A Síndrome Cri du Chat (SCDC) foi descoberta em 1963 na França pelo geneticista francês Dr. Jerone Lejeune (MACHADO et al., 2007). É uma doença genética que resulta ou de uma deleção extremamente pequena (5p15.2) ou deleção de todo o braço curto do cromossomo (5p), fazendo parte do grupo de Síndromes de supressão parcial. É uma doença rara, com uma incidência de $1 / 15000$ a 1/50000 nascidos vivos, tendo prevalência maior no sexo feminino (OVERHAUSER, J. et al., 1994; SARIMSKI, 2003; MAINARDI, et al., 2006).

A etiologia desta síndrome pode ser de origem esporádica, por uma deleção ou translocação ( $85 \%$ dos casos) ou por herança genética ( $25 \%$ restantes) $(\mathrm{CHO}$, et al., 2012), devido à uma separação desigual de uma translocação equilibrada nos cromossomos de um dos pais, fato que não gera perda de material genético, de modo que um dos pais seja apenas portador de translocação equilibrada e perfeitamente normal (OTTO et al., 2004; JONES, 1998).

As manifestações clínicas caracterizam-se por choro típico, conhecido como miado de gato (devido a alterações anatômicas da laringe e da epiglote), desvio inferior das extremidades dos lábios, ponte nasal ampla e microretrognatismo, que geram dificuldades de sucção, refluxo gastroesofágico e disposição à infecções gastrointestinais. O hipertelorismo, dobras epicânticas, orelhas com baixa implantação e face arredondada acarretam assimetria facial. Altura e peso inferior 
aos padrões e hipotonia deixam a criança com susceptibilidade à infecções respiratórias. Há também prega única nas mãos e atraso do desenvolvimento psicomotor (KAJII, T. et al., 1966; HILLS, C. et al., 2006; MACHADO et al., 2007). A hiperatividade também pode estar presente em $80 \%$ dos casos, podendo haver a agressividade correlacionada (CORNISH, K. et al., 2002; MAINARDI, 2006). Além das características próprias dessa síndrome, pode haver a presença de malformações neurológicas, renais, gastrointestinais e cardíacas associadas em 15$20 \%$ dos casos (HILLS, C. et al., 2006). Com a idade evidenciam-se novas manifestações: alongamento da face, encurtamento do filtro naso-labial, má oclusão dentária, estrabismo divergente, encurtamento mais pronunciado dos metacarpos e metatarsos, descoloração precoce do cabelo e substituição da hipotonia por hipertonia (MAINARDI, et al., 2006).

O desenvolvimento verbal é lento, apresentando maior a dificuldade na comunicação do que na compreensão, $50 \%$ destes pacientes adquirem a marcha independente aos 3 anos, 25\% conseguem pronunciar frases curtas aos 4 anos e meio, $50 \%$ aos cinco e meio e a quase totalidade aos dez anos (CORNISH, K. et al., 2002; MAINARDI, et al., 2006).

O tratamento do SCDC baseia-se nas estratégias de reabilitação precoce, em que a fisioterapia atua na prevenção das complicações e na correção das malformações (MAINARDI, et al., 2006), uma vez que, de acordo com Muniz et al (2002) e Sarimski (2003), a estimulação precoce permite melhor desenvolvimento, adaptação e aceitação da sociedade, apesar de apresentar limitações como consequência da deficiência intelectual e retardo neuropsicomotor. Dessa forma, a terapia necessita visar a função, para que haja redução das limitações funcionais, além de aprimorar o bem-estar (LOLLAR, et al., 2002).

A abordagem utilizada foi o Conceito Neuroevolutivo Bobath, que se baseia na sequência do desenvolvimento típico, tratando pacientes com lesão do Sistema Nervoso Central (RAINE, 2006; IBITA, 2011). Este conceito visa a restauração dos movimentos normais e eliminação de movimentos anormais (GUSMAN, As. et al., 2010). 
A fisioterapia permitirá a melhora desenvolvimento, adaptação e aceitação da sociedade, pois visa o aprimoramento da função, para que haja redução das limitações funcionais, além de aprimorar o bem-estar (LOLLAR, et al., 2002).

Desse modo, o objetivo deste trabalho foi verificar a eficácia da contribuição do Conceito Bobath no tratamento da paciente com Síndrome de Cri Du Chat, visto que, devido à escassez de literatura comprovando a efetividade da fisioterapia no tratamento dessa doença, justifica-se a importância deste estudo.

\section{METODOLOGIA}

O presente estudo é caracterizado por um estudo de caso realizado na Associação Norte Paranaense de Reabilitação em Maringá-PR (ANPR), os responsáveis da paciente assinarem o Termo de Consentimento Livre e Esclarecido (TCLE).

A criança recrutada é do sexo feminino, 4 anos de idade e com diagnóstico confirmado da síndrome por exame genético.

A paciente foi submetida à avaliação com a presença dos seus responsáveis e tratada em 20 sessões, durante aproximadamente 30 minutos, com repetições de 2 vezes por semana, por meio do Conceito Neuroevolutivo Bobath.

Os protocolos que foram utilizados para avaliar as variáveis desta pesquisa são o Teste Denver e Inventário de Avaliação Pediátrica de Incapacidade (PEDI). O primeiro consiste em um teste padronizado do desenvolvimento nos aspectos pessoal-social, motor-fino adaptativo, linguagem, motor amplo e comportamental (REZENDE et al., 2005; SOUZA et al., 2008; CUSTÓDIO et al., 2012). O segundo é um instrumento de avaliação infantil, que permite uma descrição do desempenho funcional da criança através do autocuidado, mobilidade e função social, além de informar sobre o grau de independência da mesma e se ela faz uso de alguma alteração no ambiente para ajudar no seu desempenho (MANCINI, 2005).

Após a realização das sessões de fisioterapia propostas, a criança foi reavaliada com os mesmos protocolos e a análise dos resultados foi através de tabelas comparativas com o antes e após o tratamento.

Fórum Internacional de Qualidade de Vida e Saúde - Curitiba, 15 de junho de 2019 


\section{RESULTADOS}

O estudo de caso foi feito com M.M.N, sexo feminino, 44,4 meses diagnosticada com SCDC aos 3 meses de vida através do cariótipo.

Durante a avaliação inicial, a paciente apresentou-se hipotônica grau leve, presença de todas as reações, ausência de todos os reflexos primitivos e babinski positivo. Apresentou déficit de equilíbrio dinâmico e marcha com apoio presente.

Foram aplicados dois testes, Denver e o PEDI, na avaliação, para verificar a presença atraso motor e depois reavaliado com os mesmos critérios da avaliação. Os dados obtidos pelo Denver estão descritos na Tabela 1 e os obtidos pelo PEDI estão na Tabela 2.

Tabela 1 - Dados obtidos através do Teste Denver na avaliação inicial e na reavaliação após 20 atendimentos.

\begin{tabular}{lll}
\hline Denver & Avaliação & Reavaliação \\
\hline Anda sozinha, raramente cai & Não & Não \\
Tira sozinha qualquer peça do vestuário & Sim & Sim \\
Combina duas ou três palavras & Não & Não \\
Distancia-se da mãe sem perde-la de vista & Sim & Sim \\
Leva alimentos à boca com a própria mão & Sim & Sim \\
Corre e/ou sobre degraus baixos & Sim, com & Sim, com \\
& apoio & apoio \\
Brinca isoladamente na presença de outras & Sim & Sim \\
crianças & & \\
$\begin{array}{l}\text { Diz seu próprio nome e nomeia objetos como } \\
\text { sendo seu }\end{array}$ & Não & Não \\
Veste-se com auxílio & & Sim \\
Fica sobre um pé momentaneamente & Não & Não \\
Usa frases & Não & Não \\
Controle esfincteriano & Não & Não \\
Reconhece mais de duas cores & Não & Sim \\
\hline
\end{tabular}




\begin{tabular}{lll}
\hline Pula sobre um pé só & Não & Não \\
Brinca com outras crianças, interagindo & Sim & Sim \\
Imita pessoas da vida cotidiana & Sim & Sim \\
Veste-se sozinha & Não & Não \\
Pula alternadamente com um e outro pé & Não & Não \\
$\begin{array}{l}\text { Alterna momentos cooperativos com agressivos } \\
\begin{array}{l}\text { Capaz de expressar preferências e ideias } \\
\text { próprias }\end{array}\end{array}$ & Sim & Sim \\
\hline
\end{tabular}

Tabela 2 - Dados obtidos através do Inventário de Avaliação Pediátrica de Incapacidade (PEDI), na avaliação inicial representados pela cor amarela e dados obtidos na reavaliação representados pela cor verde. A cor azul representa dados de respostas iguais em ambas avaliações.

\begin{tabular}{|c|c|c|c|c|c|c|c|c|c|c|}
\hline \multirow[b]{3}{*}{ Área de Auto-Cuidado } & \multicolumn{6}{|c|}{ Assistencia do Cuidados } & \multicolumn{4}{|c|}{ Modificações } \\
\hline & 5 & 4 & 3 & 2 & 1 & 0 & $\mathbf{N}$ & C & $\mathbf{R}$ & $\mathbf{E}$ \\
\hline & 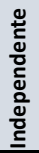 & 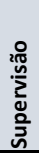 & 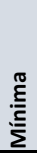 & $\begin{array}{l}\frac{\pi}{0} \\
\frac{\pi}{2} \\
\frac{\pi}{8} \\
\Sigma\end{array}$ & 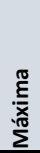 & 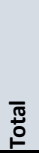 & 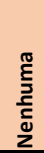 & 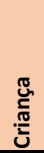 & 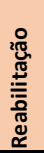 & 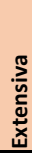 \\
\hline $\begin{array}{l}\text { A. Alimentação: Come e bebe nas refeições regulares, não inclui cortar carne, } \\
\text { abrir recipientes ou servir comida das travessas. }\end{array}$ & 5 & 4 & 3 & 2 & 1 & 0 & $\mathrm{~N}$ & C & $\mathrm{R}$ & $\mathrm{E}$ \\
\hline B. Higiene Pessoal: escova dentes, escova ou penteia o cabelo e limpa o nariz. & 5 & 4 & 3 & 2 & 1 & 0 & $\mathrm{~N}$ & C & $\mathrm{R}$ & $\mathrm{E}$ \\
\hline $\begin{array}{l}\text { C.Banho: lava e seca rosto e mãos, toma banho; não inclui: entrar e sair do } \\
\text { chuveiro ou banheira, preparar a água e lavar costas e cabelos }\end{array}$ & 5 & 4 & 3 & 2 & 1 & 0 & $\mathrm{~N}$ & C & $\mathrm{R}$ & $E$ \\
\hline $\begin{array}{l}\text { D. Vestir- parte superior do corpo: roupas de uso diário, inclui ajudar a colocar } \\
\text { e retirar splint ou prótese; não inclui: tirar roupas do armário ou gavetas, lidar } \\
\text { com fechos nas costas }\end{array}$ & 5 & 4 & 3 & 2 & 1 & 0 & $\mathrm{~N}$ & C & $\mathrm{R}$ & $E$ \\
\hline $\begin{array}{l}\text { E.Vestir- parte inferior do corpo: roupas de uso diário, incluindo colocar e tirar } \\
\text { órtese ou prótese; não inclui tirar as roupas do armário ou gavetas. }\end{array}$ & 5 & 4 & 3 & 2 & 1 & 0 & $\mathrm{~N}$ & C & $\mathrm{R}$ & $E$ \\
\hline
\end{tabular}

A intervenção foi realizada por meio dos fundamentos do Conceito Bobath, com objetivo de estimular a paciente a aprimorar o alinhamento de tronco e 0 equilíbrio dinâmico para adquirir a marcha independente.

$\mathrm{Na}$ avaliação inicial foi detectado um atraso motor em relação a idade cronológica da criança, em que a mesma se encontrava entre 9 e 10 meses. Após os atendimentos com estímulos através dos pontos-chave e exercícios terapêuticos, a paciente apresentou pequenas melhoras no equílibrio e alinhamento de tronco, porém ainda com tendência à fazer extensão de tronco ao andar, conseguindo realizar a deambulação independente por muitos passos. A seguir, uma 
representação gráfica da Idade Cronológica e Idade Motora antes e após os atendimentos.

Gráfico 1 - Idade Motora e Idade Cronológica em meses da paciente na avaliação inicial e reavaliação após 20 atendimentos.

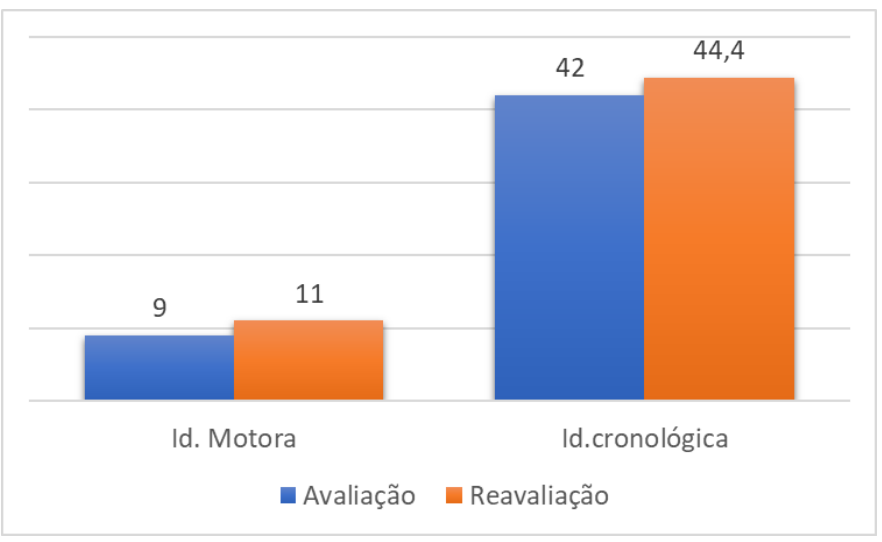

Desde $01^{\circ}$ atendimento foi-se estimulado a marcha, evoluindo para o pontochave quadril e, após, para a marcha independente. De início, a paciente não conseguia trocar 7 passos consecutivos, mas no decorrer dos atendimentos, ela conseguiu andar livremente por muitos passos sem qualquer tipo de apoio.

Também foram realizados exercícios de propriocepção, utilizando cama elástica, disco proprioceptivo, prancha de equilíbrio e bola suíça, fazendo uso bilateral de tala extensora para joelhos para a realização dos mesmos. O uso da tala se deu por motivos de a paciente realizar a flexão de joelhos quando estava em ortostatismo.

Além disso, foram-se realizados estímulos para alinhamento de tronco, com foco no deslocamento anterior de tronco, visto que a paciente tinha a tendência em fazer extensão de tronco ao deambular. A paciente fazia uso bilateral de tala extensora de joelhos, sendo estimulada a vir para a frente. No $10^{\circ}$ atendimento, foram-se retiradas totalmente as talas, pois a paciente já não fletia mais os joelhos ao ficar em pé. 


\section{DISCUSSÃO}

Segundo os relatos de Kleim et al. (2008) e Graham et al. (2009) em seus estudos, relatam que o Conceito Neuroevolutivo Bobath se baseia na capacidade do cérebro reorganizar-se após uma lesão, de modo que áreas não comprometidas passam a compensar as funções que eram realizadas pelas regiões danificadas.

De acordo com a pesquisa da Ávila et al. (2016), os exercícios para controle de cabeça e tronco são essenciais para crianças hipotônicas, uma vez que ajudam a desenvolver força muscular e equilíbrio, favorecendo os padrões motores próximos dos típicos. Este fato confirma com nosso estudo, onde também houve aprimoramento das habilidades motoras que incluem o equilíbrio e força muscular na paciente com SCDC através de exercícios de alinhamento postural e de propriocepção.

Para Howle (2002) o tratamento deve ser flexível e adaptado às necessidades do paciente e depende do resultado da avaliação funcional (DE ALCÂNTARA et al., 2011). No estudo de Mainardi (2006), a conduta fisioterapêutica consistiu-se na intervenção precoce, com foco na precaução das complicações e na correção das deformidades. Isso condiz com a conduta que foi utilizada em nossa pesquisa, na qual a paciente foi submetida a exercícios que buscavam o alinhamento de tronco, apresentando, no final mais domínio do mesmo, principalmente durante a marcha.

Em uma revisão bibliográfica sobre "Habilitação e Reabilitação: Fisioterapia aplicada em crianças com problemas neurológicos", Gusman (2010) encontrou evidências em seu estudo, em que o Conceito Neuroevolutivo Bobath tem como proposito restaurar movimentos normais e inibir movimentos atípicos. Por isso, no presente estudo, buscou-se instruí-la na realização dos movimentos típicos, iniciados pela própria paciente, com auxilio das pesquisadoras, sempre focando no desenvolvimento motor típico.

Para Hills (2006) e Simmons (1998), a criança com Síndrome de Cri Du Chat apresenta, entre outras características, altura e peso inferior à dos padrões, hipotonia, atraso do desenvolvimento psicomotor e microcefalia, que acarretam alterações significativas no sistema neuromuscular. Em função disso LOLLAR, et al., (2002) sugerem que a fisioterapia permite a melhora desenvolvimento, adaptação e Fórum Internacional de Qualidade de Vida e Saúde - Curitiba, 15 de junho de 2019 
aceitação da sociedade, pois visa o aprimoramento da função, para que haja redução das limitações funcionais, além de aprimorar o bem-estar.

A experiência em tratar a paciente foi gratificante e de muito aprendizado pessoal e profissional, visto a capacidade da mesma em evoluir no tratamento, de modo gradual, desenvolvendo coragem para realizar o que lhe fora proposto. A cada atendimento, houve desafios para deixa-la entretida e focada e para deixá-la animada em todo o decorrer do atendimento diário. Entretanto, devido à escassez de trabalhos associando o Conceito Neuroevolutivo Bobath com a Síndrome Cri Du Chat, faz-se necessário a produção de mais estudos com essa relação, para que haja maiores comprovações de sua eficácia.

\section{CONCLUSÃO}

Pode-se concluir, com o presente estudo, que o tratamento fisioterapêutico utilizando o Conceito Neuroevolutivo Bobath, foi capaz de melhorar o desenvolvimento motor da criança com Síndrome Cri Du Chat, promovendo melhora da independência da mesma, no que diz respeito à marcha e equilíbrio.

\section{REFERÊNCIAS}

AMORIM, R. F. 2011. 76 f. Trabalho de Pós-graduação - Especialização em Fundamentos Curriculares da Educação Inclusiva - Universidade do Estado de Santa Catarina, Florianópolis, 2011.

ÁVILA, Alexia de Souza Costa; ROCHA, Cristiano Andrade Quintão Coelho. Atuação fisioterapêutica em paciente com PC com tetraparesia espástica assimétrica: um estudo de caso. Revista Científica da FAMINAS, v. 10, n. 2, 2016.

$\mathrm{CHO}, \mathrm{E} . \mathrm{H}$. et al. High resolution microarray analysis in a patient with speech delay without cat-like cry. Genetic Counseling, v. 23, n. 2, p. 339, 2012.

CORNISH, Kim; BRAMBLE, D. Cri du chat syndrome: genotype-phenotype correlations and recommendations for clinical management. Developmental Medicine and Child Neurology, v. 44, n. 7, p. 494-497, 2002. 
CUSTÓDIO, Zaira Aparecida de Oliveira; CREPALDI, Maria Aparecida; CRUZ, Roberto Moraes. Desenvolvimento de Crianças Nascidas Pré-Termo Avaliado pelo Teste de Denver-II: Revisão da Produção Científica Brasileira. Psicol. Reflex. Crit. vol.25 no.2 Porto Alegre, 2012

DE ALCÂNTARA, Clarissa Byrro; COSTA, Cláudia Maria Byrro; DE LACERDA, Helenice Soares. Tratamento Neuroevolutivo-Conceito Bobath. 2011.

GRAHAM, Julie Vaughan et al. The Bobath concept in contemporary clinical practice. Topics in stroke rehabilitation, v. 16, n. 1, p. 57-68, 2009.

GUSMAN, S. A. Torre, CA. Habilitação e reabilitação. Fisioterapia aplicada em crianças com problemas neurológicos. Neurologia infantil. São Paulo, SP: Atheneu, v. 2, p. 1753-1775, 1753.

HILLS, Christine et al. Cri du chat syndrome and congenital heart disease: a review of previously reported cases and presentation of an additional 21 cases from the Pediatric Cardiac Care Consortium. Pediatrics, v. 117, n. 5, p. e924-e927, 2006.

HOWLE, Janet M. Neuro-developmental treatment approach: theoretical foundations and principles of clinical practice. NeuroDevelopmental Treatment, 2002.

International Bobath Instructors Training Association (IBITA). Disponível em: http://www.ibita.org/. Acesso em 14 de maio de 2018.

JONES, Kenneth Lyons; IKEDA, MARCOS. Padrões reconhecíveis de malformações congênitas. editora manole Itda, 1998.

KAJll, Tadashi et al. Cri du chat syndrome. Archives of disease in childhood, v. 41, n. 215, p. 97, 1966.

KLEIM, Jeffrey A.; JONES, Theresa A. Principles of experience-dependent neural plasticity: implications for rehabilitation after brain damage. Journal of speech, language, and hearing research, v. 51, n. 1, p. S225-S239, 2008.

LOLLAR, Donald J.; SIMEONSSON, Rune J. Diagnosis to function: classification for children and youths. Journal of Developmental \& Behavioral Pediatrics, v. 26, n. 4, p. 323-330, 2005.

MACHADO, NCSS et al. Principais características clínicas da síndrome Cri-Du-Chat: revisão de literatura. Encontro latino americano de iniciação científica, v. 11, p. 1668-1671, 2007.

MAINARDI, Paola Cerruti. Cri du Chat syndrome. Orphanet journal of rare diseases, v. 1, n. 1, p. 33, 2006. 
MANCINI, Marisa Cotta. Inventário da avaliação pediátrica de incapacidade (PEDI): manual da versão brasileira adaptada. In: Inventário da avaliação pediátrica de incapacidade (PEDI): manual da versão brasileira adaptada. 2005.

MUNIZ, J. W., C.; BASTOS, C. C.; AMARAL, R. O. Intervenção Fisioterapêutica na Síndrome do Cri du Chat. Lato Sensu, Belém, V. 4, n. 6, p. 112-119, nov., 2002.

OTTO, Priscila Guimarães; OTTO, Paulo Alberto; FROTA-PESSOA, Oswaldo. Genética humana e clínica. Editora Roca, 2004.

REZENDE, Magda Andrade; BETELI, Vivian César; SANTOS, Jair Lício Ferreira dos. Follow-up of the child's motor abilities in day-care centers and preschools. Revista latino-americana de enfermagem, v. 13, n. 5, p. 619-625, 2005.

SARIMSKI, K. Early play behaviour in children with $5 \mathrm{p}$-(Cri-du-Chat) syndrome. Journal of Intellectual Disability Research, v. 47, n. 2, p. 113-120, 2003.

SOUZA, Sandra Coenga de et al. Desenvolvimento de pré-escolares na educação infantil em Cuiabá, Mato Grosso, Brasil. Cadernos de Saúde Pública, v. 24, p. 1917-1926, 2008. 